\title{
Aproximación a las dificultades para la ejecución de trabajos prácticos de laboratorio de biología en educación media
}

\section{Approach to the difficulties for the execution of practical work in the biology laboratory in secondary education}

\author{
${ }^{1}$ Juan Neira Morales, ${ }^{2}$ Luis Miño González, ${ }^{3}$ Marta Fuentealba Cruz*
}

Neira, J., Miño, L., \& Fuentealba, M. (2021). Aproximación a las dificultades para la ejecución de trabajos prácticos de laboratorio de biología en educación media. Revista Convergencia Educativa, (10-extra), diciembre, 24-33.

https://doi.org/10.29035/rce.s10.24

[Recibido: 21 enero, 2021 / Aceptado: 23 septiembre, 2021]

\begin{abstract}
RESUMEN
Los trabajos prácticos de laboratorio son instancias claves en el proceso de enseñanza y aprendizaje de las ciencias biológicas y, consecuentemente, la investigación en esta temática constituye una de las líneas más importantes en la didáctica de las ciencias desde hace ya mucho tiempo, por lo que el objetivo del presente trabajo es determinar la viabilidad para el desarrollo de actividades prácticas de biología en enseñanza media. Para ello, se realizó una encuesta online a 24 profesores en ejercicio; como producto de este sondeo fueron detectadas diversas razones que dificultan la realización de dichas actividades, entre las cuales se destacan aspectos relacionados con la desmotivación de los estudiantes, falta de recursos y de espacios adecuados para realizar trabajos experimentales, así como periodos de clase muy cortos. Lo anterior conduce a advertir que, para muchos profesores, los trabajos prácticos de laboratorio han pasado a un segundo plano y se han dejado de incluir como una actividad fundamental dentro de las planificaciones del trabajo. Además, se discute la utilización de la enseñanza telemática en tiempos de pandemia por COVID-19, que nos permiten mitigar la falta de TPL tradicional, a través del diseño nuevas estrategias didácticas.
\end{abstract}

Palabras clave: Aprendizaje significativo, Estrategia didáctica, Nivel de abertura.

* Este artículo fue presentado en las jornadas Internacional de Investigación en Didáctica y Formación Inicial Docente JIIDFID_UCM 2020.

\footnotetext{
${ }^{1}$ Licenciado en Educación. Universidad Católica del Maule. Talca, Chile. https://orcid.org/0000-0002-1332-9919 | jcneira95@gmail.com

2 Doctor en Didáctica de las Ciencias Experimentales. Universidad Católica del Maule. Talca, Chile. https://orcid.org/0000-0003-3262-2034 | lmino@ucm.cl

3 Doctor en Ciencias Ambientales. Universidad Católica del Maule. Talca, Chile. https://orcid.org/0000-0003-2135-6869 | mfuentea@ucm.cl
} 
Neira, J., Contreras, P., Miño, L., \& Fuentealba, M. (2021). Aproximación a las dificultades para la ejecución de trabajos prácticos de laboratorio de biología en educación media. Revista Convergencia Educativa, (10-extra), diciembre, 24-33. https://doi.org/10.29035/rce.s10.24

\begin{abstract}
The practical laboratory works are key in the teaching and learning process of biological sciences and, consequently, research on this subject constitutes one of the most important lines in science didactics for a long time, so the aims of the present work is to determine the viability for the development of practical activities of biology in secondary education. To do this, an online survey was conducted with 24 practicing teachers. Detecting various reasons that make it difficult to carry out these activities, among which aspects related to the demotivation of students, lack of resources and adequate spaces to carry out experimental work, as well as very short class periods stand out. This leads to the belief that, for many teachers, practical laboratory work has taken a back seat and is no longer included as a fundamental activity within work schedules. In addition, the use of online teaching is discussed in times of a COVID-19 pandemic, which allows us to mitigate the lack of traditional TPL, through the design of new teaching strategies.
\end{abstract}

Key words: Meaningful learning, Didactic strategy, opening level

\title{
INTRODUCCIÓN
}

El ejercicio docente se caracteriza por utilizar variadas herramientas y estrategias, para entregar los contenidos curriculares y promover los aprendizajes entre los estudiantes (Aguilera et al., 2017, Fuentealba et at., 2017, Galagovsky, 2007, Jiménez et al., 2006, Monzon et al., 2020, Uribe et al., 2009). En Ciencias Biológicas, una de estas estrategias son los trabajos prácticos de laboratorio (TPL), que son instancias de construcción de conocimiento por sus características intrínsecas, es decir, centradas en los estudiantes, utilizan el método científico, son realizadas en un ambiente diferente al aula tradicional y, contribuyen al desarrollo de competencias actitudinales, conceptuales y procedimentales (Espinosa et al., 2016, Fernández, 2018, Quintanilla et al., 2014, Severiche \& Acevedo, 2013).

No obstante, se ha descrito que la forma en que se implementan las actividades prácticas, no incentiva la indagación en los jóvenes, haciendo que los TPL se tornen monótonos, disminuyendo el apetito cognitivo por el estudio de las ciencias (Espinosa et al., 2016, Guevara \& Lemus, 2019) debido a que, por lo general los docentes de ciencias implementan actividades prácticas de un bajo nivel de indagación científica. Los trabajos más frecuentes que se implementan son demostraciones, experimentos y en menor grado las actividades de tipo indagatorias (Crisafulli \& Villalba, 2013). Si bien se ha observado que los estudiantes valoran positivamente el trabajo práctico (Cofré et al., 2010), se ha descrito que, a menudo, los educandos no utilizan una adecuada metodología científica en el desarrollo de actividades prácticas, y en la mayoría de los casos se dedican a comprobar conceptos que han sido previamente estudiados en clases teóricas (Cofré et al., 2010, Zorrilla \& Mazzitelli, 2015, Fernández, 2018) lo que implica que esas actividades prácticas presenten bajos niveles de abertura, entendido como la cantidad de información que se les facilita a los estudiantes en una actividad práctica (Jiménez et al., 2006). Priestley (1997) basándose en trabajos previos sobre esquemas orientados a la investigación, propuso una escala de siete niveles de abertura para los trabajos prácticos de laboratorio (TPL), los que se describen en la Tabla 1. 


\section{Tabla 1}

Niveles de abertura según Priestley (1997).

\begin{tabular}{|c|c|c|c|}
\hline Nivel & Título & Descripción de las actividades en el laboratorio & $\begin{array}{l}\text { Proceso cognitivo } \\
\text { requerido }\end{array}$ \\
\hline 1 & $\begin{array}{l}\text { Herméticamente } \\
\text { cerrado }\end{array}$ & $\begin{array}{l}\text { Se proporcionan todos los procedimientos a los } \\
\text { estudiantes. Los estudiantes escriben los datos en los } \\
\text { espacios reservados de un informe de laboratorio. Se } \\
\text { incluyen tablas con los datos. }\end{array}$ & Conocimiento \\
\hline 2 & Muy cerrado & $\begin{array}{l}\text { Se proporcionan todos los procedimientos a los } \\
\text { estudiantes. Se incluyen tablas de datos }\end{array}$ & Conocimiento \\
\hline 3 & Cerrado & $\begin{array}{l}\text { Se proporcionan todos los procedimientos a los } \\
\text { estudiantes }\end{array}$ & $\begin{array}{l}\text { Conocimiento y } \\
\text { comprensión }\end{array}$ \\
\hline 4 & Entreabierto & $\begin{array}{l}\text { Se proporcionan todos los procedimientos a los } \\
\text { estudiantes. } \\
\text { Algunas preguntas o conclusiones son abiertas. }\end{array}$ & $\begin{array}{l}\text { Comprensión } \\
\text { y aplicación }\end{array}$ \\
\hline 5 & $\begin{array}{l}\text { Ligeramente } \\
\text { abierto }\end{array}$ & $\begin{array}{l}\text { Se proporcionan la mayoría de procedimientos a los } \\
\text { estudiantes y algunas preguntas o cuestiones son } \\
\text { abiertas. }\end{array}$ & Aplicación \\
\hline 6 & Abierto & $\begin{array}{l}\text { Los estudiantes desarrollan sus propios procedimientos. } \\
\text { Se } \\
\text { les proporciona una lista con el material. Muchas } \\
\text { preguntas o conclusiones son abiertas. }\end{array}$ & Análisis y síntesis \\
\hline 7 & Muy abierto & $\begin{array}{l}\text { A los estudiantes se les indica un problema que tienen } \\
\text { que } \\
\text { resolver (¡o que ellos mismos proponen!). Los } \\
\text { estudiantes } \\
\text { desarrollan el procedimiento y sacan sus propias } \\
\text { conclusiones. }\end{array}$ & Síntesis y evaluación \\
\hline
\end{tabular}

Por su parte, Meroni et al. (2015) señalaron que es transcendental el mantenimiento constante del carácter indagatorio en las clases de ciencias. Asimismo, Cofré et al. (2010), argumentaron que la enseñanza, a través de la indagación científica, debe entenderse como un ente catalizador que motive a los estudiantes a sentir interés por el estudio de las ciencias naturales, para así, contribuir al fomento del desarrollo del pensamiento científico en los jóvenes estudiantes. En ese sentido, Espinosa et al. (2016) señalaron que implementar TPL, otorga la posibilidad al docente de ser un facilitador y a la vez regular el proceso de enseñanza-aprendizaje, ya que le demanda una organización del tiempo y espacio de manera adecuada, de tal forma, que los estudiantes puedan operar, manipular, realizar montajes y manejar instrumentos; facilitando las motricidades finas y las relaciones sociales, a través del trabajo en equipo. Por lo que, los TPL como estrategia didáctica, favorecen la comunicación entre el estudiante, el docente y el contenido a tratar, permitiendo el desarrollo de habilidades investigativas como: la observación, la predicción, el planteamiento de hipótesis, la medición, entre otras. Así como también, se desarrollan destrezas de manipulación (manejo de material de laboratorio y montaje de instrumental y equipos necesarios), favoreciendo en los alumnos una actitud positiva hacia la disciplina; además, se va inculcando en los estudiantes la construcción de una imagen de ciencia desde su naturaleza, lo que resulta relevante, convirtiéndose, en una herramienta que potencializa la enseñanza y el aprendizaje de la materia (Fernández, 2013, 2018, López \& Tamayo, 2012). Aportando, además, al desarrollo de habilidades y destrezas en los estudiantes que conllevan a la formación de un pensamiento científico, crítico y reflexivo 
Neira, J., Contreras, P., Miño, L., \& Fuentealba, M. (2021). Aproximación a las dificultades para la ejecución de trabajos prácticos de laboratorio de biología en educación media. Revista Convergencia Educativa, (10-extra), diciembre, 24-33. https://doi.org/10.29035/rce.s10.24

de las ciencias y su papel en la sociedad, que están acordes a los lineamientos y a estándares curriculares (Flores et al., 2009).

De acuerdo a lo anterior el objetivo del presente trabajo es determinar la viabilidad para el desarrollo de TPL de biología en enseñanza media. Entre las preguntas de investigación a responder están: ¿Cuáles son las razones por las cuáles no se realizan actividades prácticas? ¿Existen diferencias en los niveles de abertura de las actividades prácticas según la dependencia del establecimiento educacional?

\section{METODOLOGÍA}

\section{Tipo de estudio}

Se realizó un estudio cualitativo de análisis descriptivo, en el cual se utilizó una entrevista semiestructurada implementada a través de una encuesta, seleccionando profesores de biología en ejercicio.

La entrevista semiestructurada versó en las siguientes interrogantes:

$\checkmark \quad ¿$ Qué tipo de actividades prácticas realiza en sus clases?

$\checkmark \quad$ ¿Con qué frecuencia implementa actividades prácticas en sus clases?

$\checkmark \quad$ ¿Qué recursos pedagógicos utiliza para la ejecución de las actividades prácticas?

$\checkmark \quad$ A su juicio, ¿cuáles son las principales dificultades que se presentan al realizar cada una de las actividades prácticas mencionadas previamente?

Además, los participantes entregaron información de contexto, como, el tipo de establecimiento en que ejercía, su nivel de estudios y los años de experticia profesional.

\section{Muestra}

La muestra está constituida por 24 profesores de diferente dependencia educativa de la región del Maule (Tabla 2), a quienes durante el segundo semestre de 2019 se les realizó una encuesta.

El muestreo es por conveniencia, es decir, no probabilístico, solo se consideró que todos los participantes fuesen profesores de Biología en ejercicio. 
Neira, J., Contreras, P., Miño, L., \& Fuentealba, M. (2021). Aproximación a las dificultades para la ejecución de trabajos prácticos de laboratorio de biología en educación media. Revista Convergencia Educativa, (10-extra), diciembre, 24-33. https://doi.org/10.29035/rce.s10.24

Tabla 2

Características generales de la muestra.

\section{Dependencia educativa}

Establecimientos municipales

(11)

Establecimientos subvencionados
Modalidad

Cantidad

Científico- Humanista

Técnico-profesional

Científico- Humanista

Técnico-profesional
9

2

11

2

\section{RESULTADOS}

Al consultar a los docentes qué actividades prácticas desarrollan mayormente en sus clases, el 88,2\% señaló que realizaban demostraciones y observaciones, por otra parte, el 76,4\% indicó llevar a cabo experimentos, el 35,2\% informó que trabajaban en investigaciones estructuradas, el 23,5\% notificó investigaciones abiertas y solo un $11,7 \%$ señaló elaborar proyectos de divulgación científica.

Los recursos pedagógicos más utilizados por los docentes durante la implementación de las actividades prácticas son los textos escolares y las guías; el 100\% de los profesores consultados señaló emplear textos de estudio, el 94,1\% indicó el uso de guía y sólo el 17,6\% reveló incorporar los laboratorios de computación para realizar actividades demostrativas (Tabla 3).

Tabla. 3

Recursos pedagógicos implementados por los docentes durante la implementación de actividades prácticas.

\begin{tabular}{lc} 
Recursos pedagógicos & Porcentaje \\
\hline Textos del estudiante & $100,0 \%$ \\
\hline Guías & $94,1 \%$ \\
\hline Insumos de laboratorio & $76,4 \%$ \\
\hline Manuales de laboratorio & $41,2 \%$ \\
\hline Laboratorios de computación & $17,6 \%$ \\
\hline
\end{tabular}

En relación a la frecuencia de implementación de actividades prácticas, en los establecimientos científico humanista es de 1 a 3 veces por semestre, a diferencia de los establecimientos técnico profesional donde los estudiantes tienen actividades prácticas todas las semanas. Esta frecuencia tiene relación con el nivel de abertura de las actividades prácticas la cual es mayor en los establecimientos técnico profesional (Tabla 4). 
Neira, J., Contreras, P., Miño, L., \& Fuentealba, M. (2021). Aproximación a las dificultades para la ejecución de trabajos prácticos de laboratorio de biología en educación media. Revista Convergencia Educativa, (10-extra), diciembre, 24-33. https://doi.org/10.29035/rce.s10.24

Tabla 4

Frecuencia de realización de actividades prácticas y niveles de abertura en establecimientos educacionales de diferentes dependencias educativas.

\begin{tabular}{cccc}
$\begin{array}{c}\text { Dependencia } \\
\text { educativa }\end{array}$ & Modalidad & $\begin{array}{c}\text { Frecuencia de realización de actividades } \\
\text { prácticas }\end{array}$ & $\begin{array}{c}\text { Nivel de } \\
\text { abertura }\end{array}$ \\
\hline $\begin{array}{c}\text { Establecimientos } \\
\text { municipales }\end{array}$ & Científico - Humanista & 1 a 3 veces por semestre & 3 \\
\cline { 2 - 4 } $\begin{array}{c}\text { Técnico - profesional } \\
\text { subvencionados }\end{array}$ & Científico - Humanista & 1 vez a la semana & 5 \\
\cline { 2 - 4 } & Técnico - profesional & 1 a 3 veces por semestre & 5 \\
\hline
\end{tabular}

Entre las dificultades observadas por los docentes para la implementación de actividades prácticas, se destacan la insuficiente motivación por parte de los estudiantes, carencia de recursos e infraestructura, además de la disminuida importancia otorgada por el establecimiento a las actividades prácticas (Tabla 5).

Tabla. 5

Dificultades observadas por los profesores al implementar actividades prácticas.

\begin{tabular}{lc} 
Dificultades observadas & Porcentaje \\
\hline Desmotivación por parte del estudiantado & $64,7 \%$ \\
\hline Falta de tiempo & $44,1 \%$ \\
\hline Falta de infraestructura & $47,1 \%$ \\
\hline $\begin{array}{l}\text { Baja importancia otorgada por el establecimiento a las actividades } \\
\text { prácticas }\end{array}$ & $47,1 \%$ \\
\hline Número excesivo de estudiantes & $35,3 \%$ \\
\hline
\end{tabular}

\section{DISCUSIÓN}

La enseñanza de las ciencias incorpora diversas dimensiones del contenido, entre ellas conceptuales (considerando teorías, postulados, conceptos, principios, leyes y lenguaje propio de la disciplina), procedimentales y actitudinales (forma de percibir, valorar y asumir la ciencia) (Carp et al., 2012, Carrascosa et al., 2006, Fernández, 2013, Fernández, 2018), siendo los TPL excelentes estrategias didácticas para el aprendizaje de muchos de estos contenidos, sin embargo, la baja frecuencia de su realización y el uso tradicional del laboratorio, donde el estudiante reproduce protocolos, disminuye la eficiencia y eficacia de esta estrategia didáctica (Merino \& Herrero, 2007), restando la posibilidad de generación de construcción de nuevos conocimientos en los estudiantes (Carp et al., 2012, Espinosa et al., 2016, Landau et al., 1997). Los TPL representan mucho más que un apoyo para las clases teóricas; su papel es importante en cuanto despierta y desarrolla la curiosidad de los estudiantes, asistiéndolos en la resolución de problemas, la explicación y comprensión de los fenómenos con los cuales interactúan en su cotidianeidad (López \& Tamayo, 2012, Severiche \& Acevedo, 2013). 
Neira, J., Contreras, P., Miño, L., \& Fuentealba, M. (2021). Aproximación a las dificultades para la ejecución de trabajos prácticos de laboratorio de biología en educación media. Revista Convergencia Educativa, (10-extra), diciembre, 24-33. https://doi.org/10.29035/rce.s10.24

Los resultados de las encuestas del presente trabajo, nos indican que antes de la pandemia los TPL en biología se realizaban con baja frecuencia y con bajo nivel de abertura, lo que es preocupante, dado que una clase teórica de biología, junto con la enseñanza experimental, a través de TPL creativos y continuos, puede aportar al desarrollo en los estudiantes de habilidades que exige la construcción de conocimiento científico (Carrascosa et al., 2006, Fernández, 2018, Flores et al., 2009). Por otro lado, se detectó una tendencia positiva entre el nivel de abertura y la frecuencia de ejecución de los TPL. De acuerdo a lo anterior, estamos ante un gran desafío, ya que tenemos necesidad de incrementar la frecuencia de TPL, pero estamos frente a un escenario incierto de salud pública; que nos obliga a tomar medidas preventivas ajenas a nuestra cotidianeidad, como la distancia física, lo que nos ha conducido a la enseñanza telemática, donde los TPL tradicionales no tienen cabida. Por lo que cabe preguntarse ¿Qué estrategia didáctica podría reemplazar a los TPL durante el contexto de pandemia COVID-19? ¿Cuáles son los desafíos para la incorporación de TPL en la enseñanza de la biología en educación media post-pandemia COVID-19? Sin dudas como lo señalan Begoña (2004) García (2017) y Madariaga et al. (2020), la educación virtual a distancia es una oportunidad para eliminar barreras espaciotemporales en el proceso educativo, donde el trabajo colaborativo e interdisciplinario son fundamentales.

Por lo que, frente a este gran reto y considerando que la enseñanza telemática existía antes de la pandemia (Begoña, 2004, Clares, 2000, Páez 2008, Pérez, 2009), debemos acoger esta oportunidad y suplir esta deficiencia que nos presenta la enseñanza remota, ya sea en su forma sincrónica como asincrónica, a través del diseño nuevas estrategias didácticas que nos permitan mitigar la falta de TPL tradicional. Lo que nos lleva a promover la realización de simulaciones prácticas de laboratorio a través de cápsulas y/o videos educativos, que aborden los contenidos propios de la asignatura, privilegiando actividades demostrativas que sean ejecutadas por el profesor y que sirvan como complemento visual al quehacer de la actividad curricular, como también, proponer actividades experimentales, que los estudiantes puedan replicar en sus hogares, y que tengan variados niveles de abertura con el fin de estimular el trabajo autónomo para favorecer el desarrollo de las habilidades científicas y del pensamiento crítico.

\section{CONCLUSIONES}

El nivel de abertura de los trabajos prácticos tiene relación con la frecuencia de éstos y es mayor en los establecimientos de educación técnico profesional. La falta de recursos y espacios adecuados para realizar trabajo experimental, así como periodos de clase muy cortos, son algunas de las razones que conducen a pensar que, en la actualidad, la actividad experimental ha pasado a un segundo plano y se ha dejado de incluir trabajo práctico en el aula, lo que se acrecentó en el año 2020 en el contexto de pandemia COVID-19. 
Neira, J., Contreras, P., Miño, L., \& Fuentealba, M. (2021). Aproximación a las dificultades para la ejecución de trabajos prácticos de laboratorio de biología en educación media. Revista Convergencia Educativa, (10-extra), diciembre, 24-33. https://doi.org/10.29035/rce.s10.24

\section{REFERENCIAS BIBLIOGRÁFICAS}

Aguilera, C., \& Manzano, A., Martínez. I., Lozano, M. \& Casiano, C. (2017). El modelo flipped classroom. International Journal of Developmental and Educational Psychology, 4(1), 261-266. https://doi.org/10.17060/ijodaep.2017.n1.v4.1055

Begoña, M. (2004). Educación y nuevas tecnologías. Educación a Distancia y Educación Virtual. Revista de Teoría y Didáctica de las Ciencias Sociales, (9), 209-222. https://www.redalyc.org/pdf/652/65200912.pdf

Carp, D., García, D., \& Chiacchiarini, P. (2012). Trabajos prácticos de laboratorio sin receta de cocina en cursos masivos. Avances en Ciencias e Ingeniería, 3(1), 167-173. https://www.redalyc.org/pdf/3236/323627685016.pdf

Carrascosa, J., Gil-Perez, D., Vilches, A., \& Valdez, P. (2006). Papel de la actividad experimental en la educación científica. Caderno Brasileiro de Ensino de Física, 23(2), 157-181. https://doi.org/10.5007/\%25x

Clares, J. (2000). Telemática, enseñanza y ambientes virtuales colaborativos. Comunicar, 7(14), 191-199. https://www.revistacomunicar.com/ojs/index.php/comunicar/article/view/C14-2000-25

Cofré, H., Camacho, J., Galaz, A., Jiménez, J., Santibáñez, D., \& Vergara, C. (2010). La Educación Científica en Chile: Debilidades de la Enseñanza y Futuros desafíos de la Educación de profesores de Ciencia. Estudios pedagógicos, 36(2), 279-293. http://dx.doi.org/10.4067/S0718-07052010000200016

Crisafulli, F., \& Villalba, H. (2013). Laboratorios para la enseñanza de las ciencias naturales en la educación media general. Educere: Revista Venezolana de Educación, 17(58), 475-485. http://www.saber.ula.ve/handle/123456789/38389

Espinosa, E., González, K., \& Hernández, L. (2016). Las prácticas de laboratorio: una estrategia didáctica en la construcción de conocimiento científico escolar. Entramado, 12(1), 266-281. https://doi.org/10.18041/entramado.2016v12n1.23125

Fernández, N. (2013). Los Trabajos Prácticos de Laboratorio por investigación en la enseñanza de la Biología. Revista de Educación en Biología, 16(2), 15-30. https://revistas.unc.edu.ar/index.php/revistaadbia/article/view/22395

Fernandez, N. (2018). Actividades prácticas de laboratorio e indagación en el aula. Tecné, Episteme y Didaxis: TED, (44), 203-218. https://doi.org/10.17227/ted.num44-9001

Flores, J., Caballero, M., \& Moreira, M (2009). El laboratorio en la enseñanza de las ciencias: Una visión integral en este complejo ambiente de aprendizaje. Revista de Investigación, 33(68), 75-111. https://revistas.upel.edu.ve/index.php/revinvest/article/view/3839 
Neira, J., Contreras, P., Miño, L., \& Fuentealba, M. (2021). Aproximación a las dificultades para la ejecución de trabajos prácticos de laboratorio de biología en educación media. Revista Convergencia Educativa, (10-extra), diciembre, 24-33. https://doi.org/10.29035/rce.s10.24

Fuentealba, M., Marín, F., Castillo, F., \& Roco, L. (2017). Análisis de la experiencia pedagógica: campamento EXPLORA Chile VA! Valorando la Biodiversidad Maulina. Revista Actualidades Investigativas en Educación, 17(1), 1-25. https://doi.org/10.15517/AIE.v17i1.27211

Galagovsky, L. (2007). Enseñanza vs. Aprendizaje de las ciencias naturales: el papel de los lenguajes y su impacto en la comunicación entre estudiantes y docentes. Revista Episteme, Tecné y Didaxis, (extra), 66-87.

García, L. (2017). Educación a distancia y virtual: calidad, disrución, aprendizajes adaptativo y móvil. RIED. Revista Iberoamericana de Educación a Distancia, 20(2), 9-25. http://dx.doi.org/10.5944/ried.20.2.18737

Guevara, M., \& Lemus, M. (2019). Las jornadas científicas fortalecen la enseñanza-aprendizaje de aspectos biológicos en educación primaria y secundaria. Revista Caribeña de Investigación Educativa (RECIE), 3(1), 95-101. https://doi.org/10.32541/recie.2019.v3i1.pp95-101

Jiménez, G., Llobera, R., \& Llitjós, A. (2006). La atención a la diversidad en las prácticas de laboratorio de Química: Niveles de abertura. Enseñanza de Las Ciencias, 24(1), 59-70. https://www.raco.cat/index.php/Ensenanza/article/view/73532/84740

Landau, L., Sileo, M., \& Lastres, L. (1997). Transformación de un trabajo práctico tradicional. Educación Química, 8(4), 200-204. http://dx.doi.org/10.22201/fq.18708404e.1997.4.66598

Lopez, A., \& Tamayo, O. (2012). Las prácticas de laboratorio en el enseñanza de las ciencias naturales. Revista Latinoamericana de Estudios Educativos, 8(1),145-166. https://www.redalyc.org/pdf/1341/134129256008.pdf

Madariaga, F., Contreras, C., Arriagada, C., Sepúlveda, M., \& Morales, K. (2020). Educación a distancia virtual ¿una oportunidad? Experiencias y desafios desde la pandemia del COVID-19. Revista Convergencia Educativa, (8), 65-82. https://doi.org/10.29035/rce.8.65

Merino, J., \& Herrero, F. (2007). Resolución de problemas experimentales de Química: una alternativa a las prácticas tradicionales. Revista Electrónica de Enseñanza de las Ciencias, 6(3), 630-648. http://reec.uvigo.es/volumenes/volumen6/ART9_Vol6_N3.pdf

Meroni, G., Copello, M., \& Paredes, J. (2015). Enseñar química en contexto. Una dimensión de la innovación didáctica en educación secundaria. Educacion Quimica, 26(4), 275-280. https://doi.org/10.1016/j.eq.2015.07.002

Monzón, V., Garrido, R., Araujo, R., \& Fuentealba, M. (2020). Promoción del conocimiento y valoración de la apidofauna nativa a través de una App Android, una experiencia didáctica. Estudios Pedagógicos, 46(2), 381-395. https://doi.org/10.4067/S0718-07052020000200381

Páez, H. (2008). Pensamiento crítico en el foro electrónico de discusión. Investigación y Postgrado, 23(2), 145-174. http://ve.scielo.org/scielo.php?script=sci_arttext\&pid=S1316-00872008000200006 
Neira, J., Contreras, P., Miño, L., \& Fuentealba, M. (2021). Aproximación a las dificultades para la ejecución de trabajos prácticos de laboratorio de biología en educación media. Revista Convergencia Educativa, (10-extra), diciembre, 24-33. https://doi.org/10.29035/rce.s10.24

Pérez, M. (2009). La comunicación y la interacción en contextos virtuales de aprendizaje. Apertura, 1(1),117. http://www.udgvirtual.udg.mx/apertura/index.php/apertura/article/view/15

Priestley, J. (1997). The impact of longer term intervention on reforming physical science teachers' approaches to laboratory instruction: Seeking a more effective role for the laboratory in science instruction [Tesis Doctoral, Temple University]. https://www.proquest.com/docview/304374092

Quintanilla, M., Joglar, C., Labarrere, A., Merino, C., Cuellar, L., \& Koponen, I. (2014). ¿Qué piensan los profesores de química en ejercicio acerca de la resolución de problemas científicos escolares y sobre las competencias de pensamiento científico?. Estudios Pedagogicos, 40(2), 283-302. http://dx.doi.org/10.4067/S0718-07052014000300017

Severiche, C., \& Acevedo, R. (2013). Las prácticas de laboratorio en las ciencias ambientales. Revista Virtual Universidad Católica Del Norte, 191-203. https://revistavirtual.ucn.edu.co/index.php/RevistaUCN/article/view/456

Uribe R., Labarrere, A., \& Santos, M. (2009). Competencias de pensamiento científico y resolución de problemas de genética simulados computacionalmente. Su contribución al aprendizaje de la biología. Enseñanza de las ciencias: revista de investigación y experiencias didácticas, (Extra), 15151520. https://raco.cat/index.php/Ensenanza/article/view/294039

Zorrilla, E., \& Mazzitelli, C. (2015). Una propuesta de Trabajos Prácticos de Laboratorio en Física para el ingreso en carreras de formación docente: Asociación de Profesores de Física de la Argentina; $\begin{array}{llllll}\text { Revista de Enseñanza de } & \text { 7a }\end{array}$ https://revistas.unc.edu.ar/index.php/revistaEF/article/view/12725

\section{Datos de correspondencia}

Marta Fuentealba Cruz

Doctor en Ciencias Ambientales

Universidad Católica del Maule. Talca, Chile.

Dirección postal: Avenida San Miguel \#3605. Talca, Chile.

ORCID ID: https://orcid.org/0000-0003-2135-6869

Email: mfuentea@ucm.cl

Esta obra está bajo una Licencia de Creative Commons Reconocimiento-NoComercial. Compartirlgual 4.0 Internacional. 